Supporting Information:

\title{
Genetically encoded protein tyrosine nitration in mammalian cells
}

Joseph J. Porter, Hyo Sang Jang, Elise M. Van Fossen, Duy P. Nguyen, Taylor S. Willi, Richard B. Cooley, Ryan A. Mehl*

Department of Biochemistry and Biophysics, 2135 ALS, Oregon State University, Corvallis OR 97331-7305 Telephone: (541) 737-4429 Fax: (541) 737-0481 Email:

ryan.mehl@oregonstate.edu

\begin{tabular}{|c|c|}
\hline \multicolumn{2}{|l|}{$\begin{array}{l}\text { Supporting Information } \\
\text { Table of Contents }\end{array}$} \\
\hline Sequences of $M$. barkeri 3-nitrotyrosine synthetase hits & $\mathrm{S} 2$ \\
\hline Media used in selections and expressions & $\mathrm{S} 2$ \\
\hline DNA oligomers used to construct eukaryotic expression vectors & S3 \\
\hline Results of initial screening of synthetase hits from library selection & S4 \\
\hline TAG suppression by the F4 synthetase/tRNA pair in HEK293T cells & S5 \\
\hline $\begin{array}{l}\text { Verification of accurate incorporation of nitroTyr and 3-nitroPhe by the } \mathrm{F} 4 \text { and A7 } \\
\text { synthetases in E. coli }\end{array}$ & S6 \\
\hline $\begin{array}{l}\text { Evaluation of nitroTyr antibodies for detecting nitroTyr and 3-nitroPhe containing } \\
\text { proteins in western blot analysis }\end{array}$ & S7 \\
\hline HEK293T cell viability assay with nitroTyr & S8 \\
\hline pAcBac1 expression vector maps & S9 \\
\hline $\begin{array}{l}\text { Flow cytometry analysis of TAG suppression in eukaryotic cells varying the ratio } \\
\text { of tRNA to synthetase }\end{array}$ & S10 \\
\hline $\begin{array}{l}\text { Structure of 14-3-3 highlighting key interactions potentially modulated by nitration } \\
\text { at site } 130\end{array}$ & S11 \\
\hline Full in gel fluorescence image of data from Figure $5 \mathrm{C}$ & S12 \\
\hline Full Coomassie and western blot images of data from Figure 5D & S13 \\
\hline
\end{tabular}


Supporting Table 1. Sequences of $M$. barkeri 3-nitrotyrosine synthetase hits.

\begin{tabular}{|ccccccc|}
\hline Mb Pyl RS hit & Leu270 & Tyr271 & Leu274 & Asn311 & Cys313 & Off Site \\
\hline D1 & Leu & Tyr & Leu & Ser & Cys & I215V/I285V \\
B2 & Leu & Tyr & Leu & Ser & Cys & \\
C2 & Leu & Tyr & Leu & Ser & Ala & \\
B3 & Phe & Tyr & Leu & Ser & Cys & E330G \\
E3 & Leu & Tyr & Leu & Ser & Cys & N401T \\
H3 & Leu & Tyr & Leu & Cys & Ala & \\
A4 & Leu & Tyr & Leu & Ser & Ser & \\
D4 & Leu & Tyr & Leu & Cys & Cys & \\
E4 & Phe & Tyr & Leu & Cys & Cys & T269A \\
F4 & Leu & Tyr & Leu & Ser & Ser & \\
G4 & Leu & Tyr & Leu & Cys & Ser & \\
C5 & Leu & Tyr & Leu & Gly & Ser & \\
B6 & Gln & Tyr & Leu & Gly & Thr & \\
E6 & Leu & Tyr & Leu & Gly & Thr & \\
\hline A7 & Leu & Tyr & Arg & Gly & Asn & P186T \\
\hline
\end{tabular}

Supporting Table 2. Media used in selections and expressions.

\begin{tabular}{|l|l|l|}
\hline \hline & A) Autoinducing Media & B) Noninducing Media \\
\hline $5 \%$ Aspartate, $\mathrm{pH} 7.5$ & $25 \mathrm{ml}$ & $25 \mathrm{ml}$ \\
\hline $10 \%$ Glycerol & $25 \mathrm{ml}$ & --- \\
\hline $25 \times 18$ amino acid mix & $20 \mathrm{ml}$ & $20 \mathrm{ml}$ \\
\hline $25 \times$ M-salts & $20 \mathrm{ml}$ & $20 \mathrm{ml}$ \\
\hline Leucine $(4 \mathrm{mg} / \mathrm{ml}), \mathrm{pH} 7.5$ & $5 \mathrm{ml}$ & $5 \mathrm{ml}$ \\
\hline $20 \%$ Arabinose $(\mathrm{w} / \mathrm{v})$ & $1.25 \mathrm{ml}$ & --- \\
\hline $1 \mathrm{M} \mathrm{MgSO} 4$ & $1 \mathrm{ml}$ & $1 \mathrm{ml}$ \\
\hline $40 \%$ Glucose & $625 \mathrm{ul}$ & $6.25 \mathrm{ml}$ \\
\hline Trace Metals & $100 \mathrm{ul}$ & $100 \mathrm{ul}$ \\
\hline & \multicolumn{2}{|c|}{ Fill to $500 \mathrm{~mL}$ with sterile water } \\
\hline
\end{tabular}


Supporting Table 3. DNA oligomers used to construct eukaryotic expression vectors.

\begin{tabular}{|c|c|}
\hline & Primer Sequence \\
\hline pJET1.2 for & 5' CGACTCACTATAGGGAGAGCGGC 3' \\
\hline P194T for & 5' CGGGAGCTGGAAACCGAGCTGGTGA 3' \\
\hline P194T rev & 5' TCACCAGCTCGGTTTCCAGCTCCCG 3' \\
\hline L282R for & 5' CTGTACAACTACAGGCGGAAACTGG 3' \\
\hline L282R rev & 5' CCAGTTTCCGCCTGTAGTTGTACAG 3' \\
\hline $\begin{array}{l}\mathrm{N} 319 \mathrm{G} / \mathrm{C} 321 \mathrm{~N} \\
\text { for }\end{array}$ & 5' GTTTACAATGGTGGGCTTTAACCAGATGGGCAGCGG 3' \\
\hline $\begin{array}{c}\mathrm{N} 319 \mathrm{G} / \mathrm{C} 321 \mathrm{~N} \\
\mathrm{rev}\end{array}$ & 5' CCGCTGCCCATCTGGTTAAAGCCCACCATTGTAAAC 3' \\
\hline pylRS rev & 5' GACTCGAGCGGCCGCCACTGTG 3' \\
\hline JP1 & $\begin{array}{l}\text { 5'- } \\
\text { TAAACTTgctagcgccGCCACCATGGACTACAAGGACGACGACGACAA } \\
\text { GG } \\
\text { ACAAGAAA-3' }\end{array}$ \\
\hline JP2 & 5'-caggtagttgtaAAAGGCgggggccagc-3' \\
\hline JP3 & 5'-gctggccccGCCTTTtacaactacctg-3' \\
\hline JP4 & 5'-cccatctggcaaaaGCAcaccattgtaaactc-3' \\
\hline JP5 & 5'-gagtttacaatggtgTGCttttgccagatggg-3' \\
\hline JP6 & $\begin{array}{l}\text { ' }- \\
\text { CAGCGGGTTTAAACGGGCCCTCTAGTCATCACAGGTTGGTGCTGAT } \\
\text { CGTTGTAGTAGC-3' }\end{array}$ \\
\hline JP7 & $\begin{array}{l}\text { 5’- } \\
\text { CTGTGTGCTAGCgccgccaccATGGTTTCTAAAGGTGAAGAACTTTTTA } \\
\text { CTGG-3' }\end{array}$ \\
\hline JP8 & $\begin{array}{l}\text { 5'- } \\
\text { ctgcaaGAATTCTTAGTGGTGATGGTGGTGATGAGTAGAATCCAGTCC } \\
\text { C-3' }\end{array}$ \\
\hline JP9 & $\begin{array}{l}\text { '- } \\
\text { TAAACTTgctagcGCCACCATGGCGTGTCCGGTTCCTTTGCAGTTGCC } \\
\text { TCC-3' }\end{array}$ \\
\hline JP10 & $\begin{array}{l}\text { 5'- } \\
\text { AGTggagaattcTCATCACAGGTTGGTGCTGATGCCGTTGTAGTAGCTC } \\
\text { TCGC-3' }\end{array}$ \\
\hline
\end{tabular}




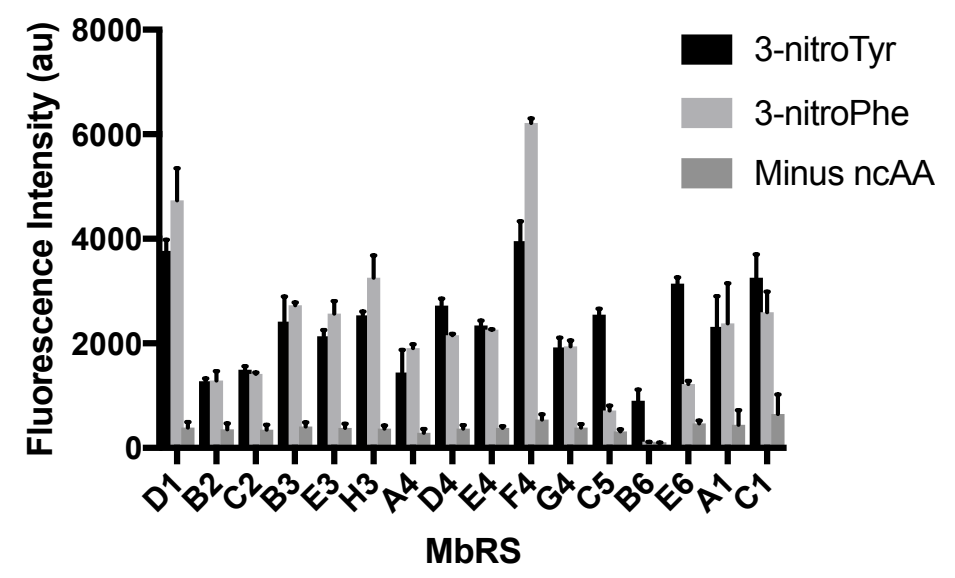

Supporting Figure 1. Results of initial screening of synthetase hits from library selection. Assessment of fluorescence from culture expressing the sfGFP150TAG gene along with each of the 14 unique $M$. barkeri pyrrolysyl synthetase variants identified from the selection process. Cultures were expressed in the presence of $1 \mathrm{mM} 3$-nitroTyr (black), $1 \mathrm{mM}$ 3-nitroPhe (light grey), or in the absence of ncAA (dark grey). 


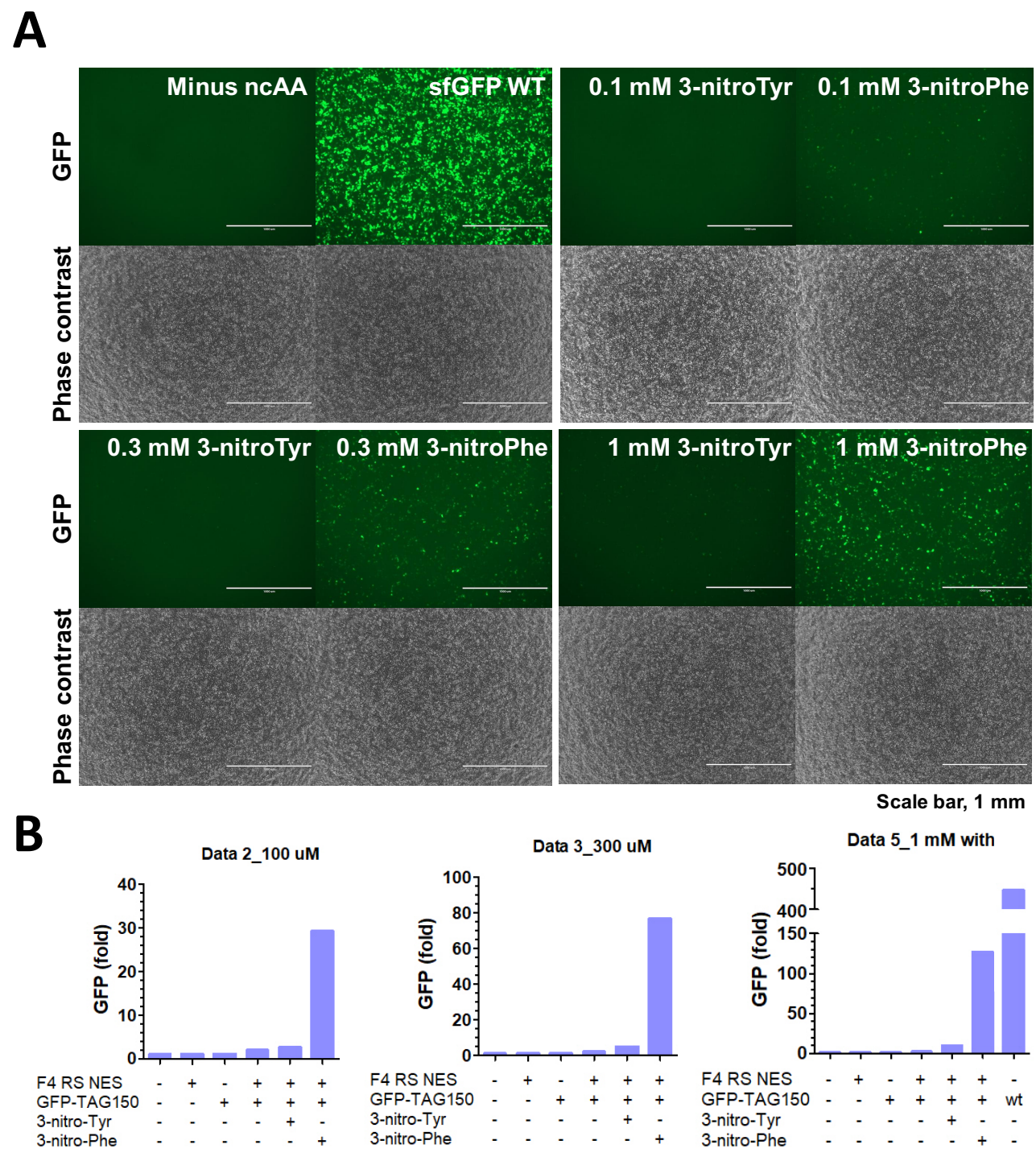

Supporting Figure 2. TAG suppression by the F4 synthetase/tRNA pair in HEK293T cells. (A) HEK293T cells transfected for 48 hours with sfGFP-150TAG pAcBac1 and F4 synthetase/tRNA in media supplemented with the indicated amount of ncAA were imaged on an EVOS FL imaging system. In the panel labeled sfGFP WT, a pAcBacl containing the WT sfGFP gene was used in place of the sfGFP-150TAG pAcBac1. (B) The relative level of sfGFP fluorescence at each concentration of ncAA is quantified based on flow cytometry data. 

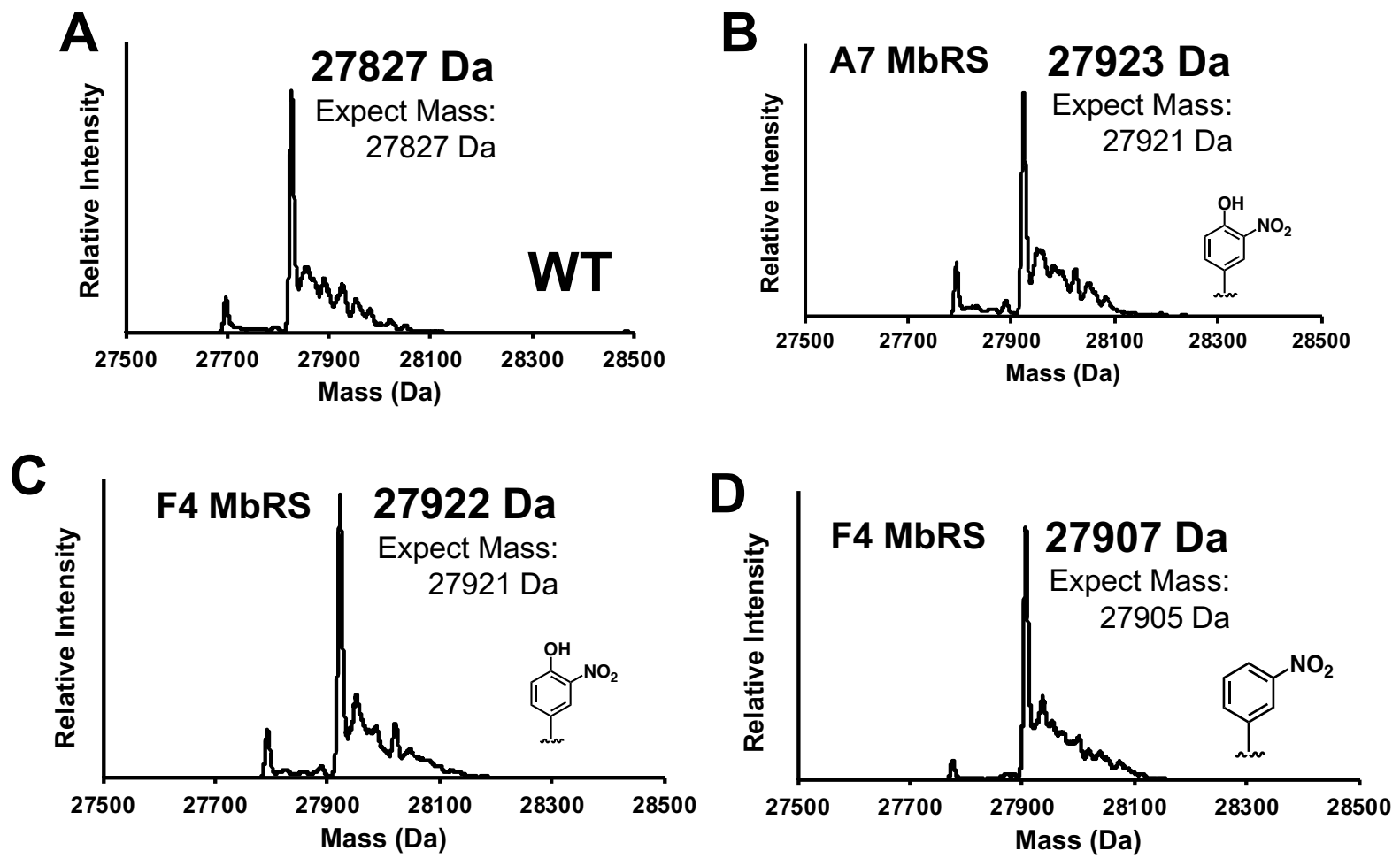

Supporting Figure 3. Verification of accurate incorporation of nitroTyr and 3-nitroPhe by the F4 and A7 synthetases in E. coli.

(A-D) Deconvoluted ESI mass spectra of purified sfGFP150 variants shown in Figure 1C. The amino acid incorporated is indicated in the lower right corner and the synthetase used in indicated in the upper left corner of each panel. Peaks corresponding to the loss of the N-terminal methionine and sodium adducts are present in the mass spectra. 

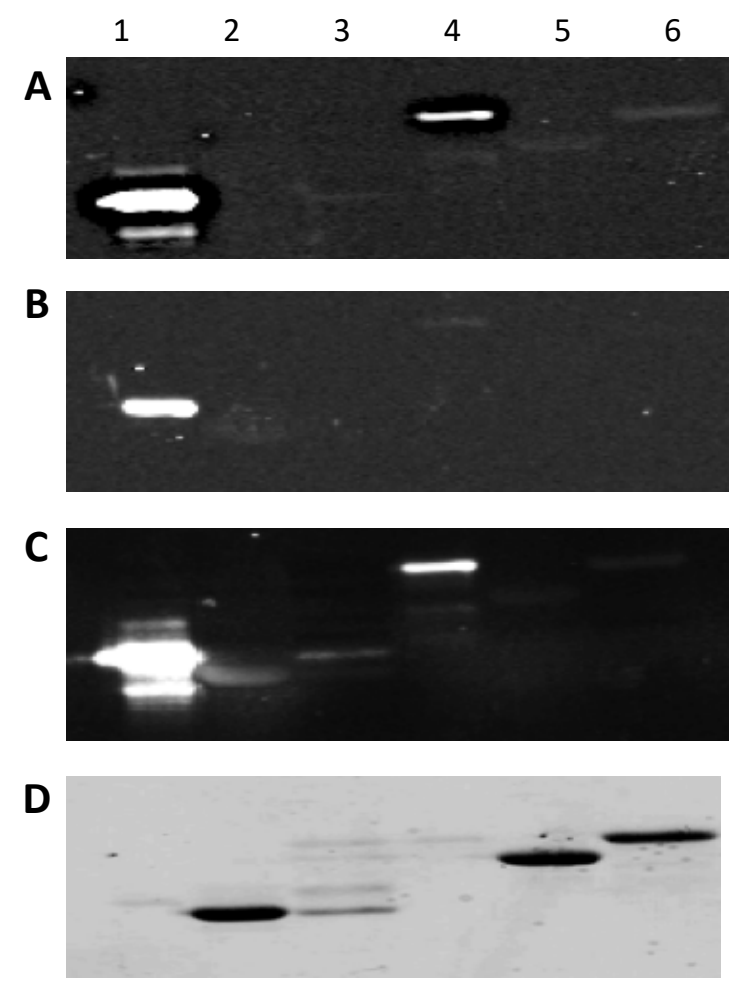

Supporting Figure 4. Evaluation of nitroTyr antibodies for detecting nitroTyr and 3-nitroPhe containing proteins in western blot analysis.

Immunoblots were probed with (A) Cayman polyclonal nitroTyr antibody, (B) Millipore monoclonal 1A6 nitroTyr antibody, (C) Millipore polyclonal antibody, and (D) a Coomassie stained gel was used as a loading control. Lane 1: $50 \mathrm{ng}$ of purified MnSOD with nitroTyr incorporated at site 34. Lane 2:1 $\mu \mathrm{g}$ of purified WT-MnSOD. Lane 3: $100 \mathrm{ng}$ of purified MnSOD with 3-nitroPhe incorporated at site 34. Lane 4: $50 \mathrm{ng}$ of human $1433 \beta$ purified from $E$. coli with nitroTyr incorporated at site 130. Lane 5: $1 \mu \mathrm{g}$ of human WT-1433 $\beta$ purified from E. coli. Lane 6: $1 \mu \mathrm{g}$ of human $1433 \beta$ purified from E. coli with 3nitroPhe incorporated at site 130 . 
ncAA effect on viability

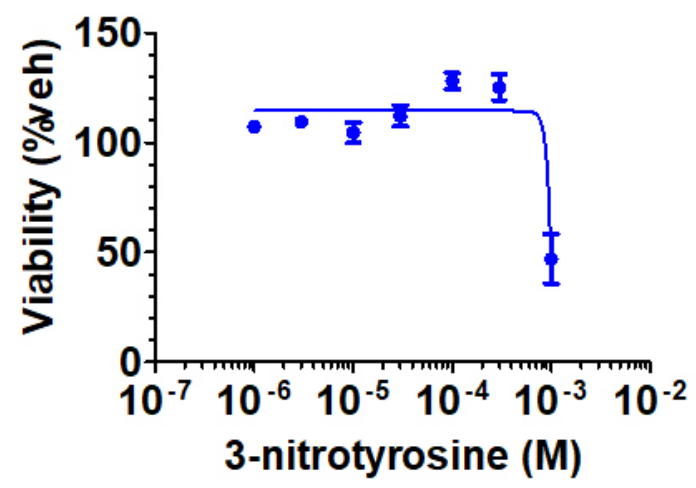

Supporting Figure 5. HEK293T cell viability assay with nitroTyr.

HEK293T cells were incubated for $48 \mathrm{~h}$ with nitroTyr and the cell viability was measured using CellTiter Glo assay kit (Promega). The data was normalized to vehicle control and fitted to a curve using non-linear regression method using GraphPad Prism 5. Based on these results the media in which HEK cells are grown can be supplemented up to $0.3 \mathrm{mM}$ with nitroTyr without significant toxicity. 

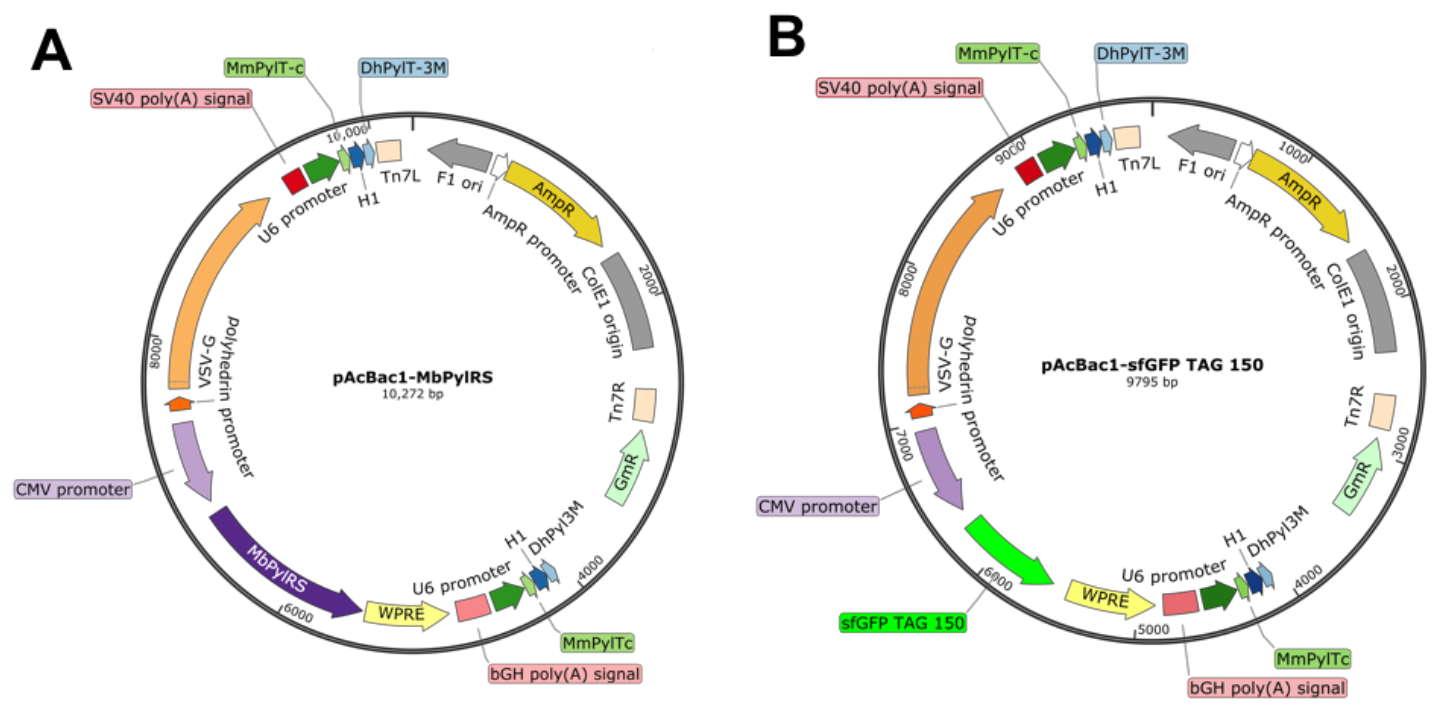

Supporting Figure 6. pAcBac1 expression vector maps.

(A) pAcBac1-MbPylRS. (B) pAcBac1-sfGFP TAG 150. 
$\mathbf{A}$

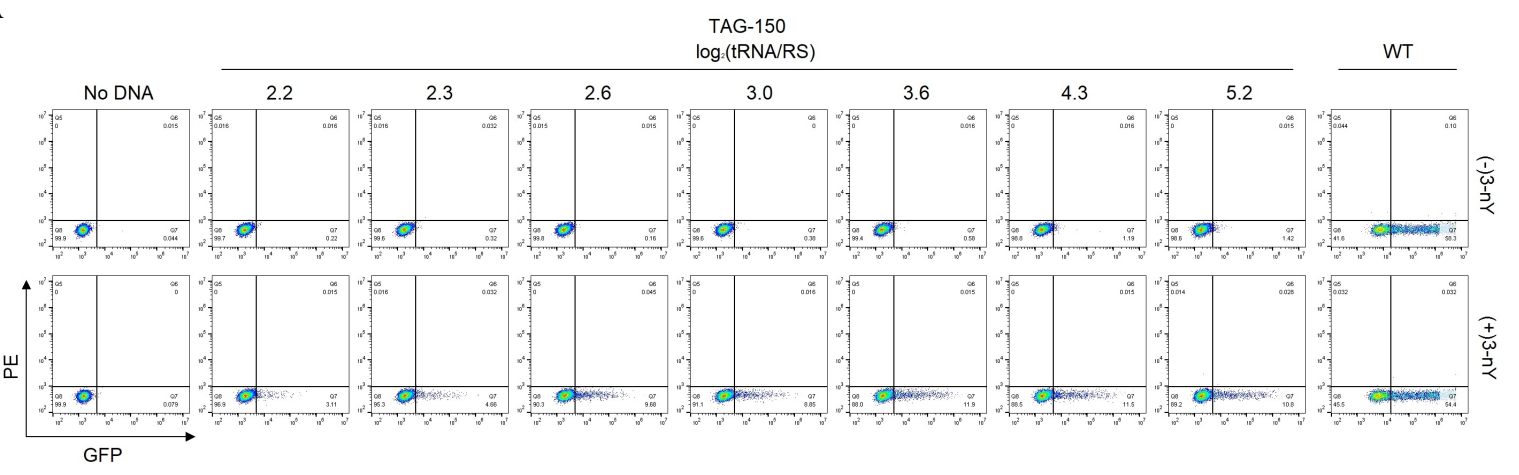

B

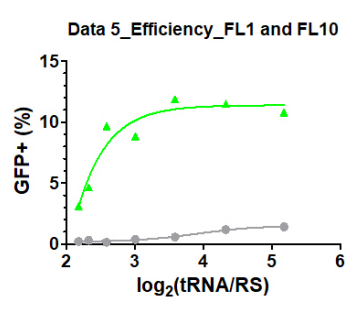

C

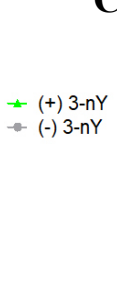

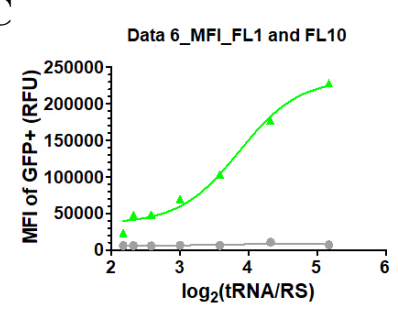

D

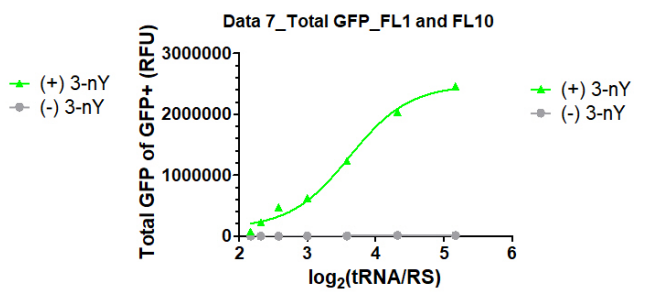

Supporting Figure 7. Flow cytometry analysis of TAG suppression in eukaryotic cells varying the ratio of tRNA to synthetase.

(A) HEK293T cells transfected with varying ratios of A7 synthetase/tRNA and sfGFP-150TAG pAcBac1 were analyzed by flow cytometry. The GFP axis shows GFP fluorescence and the PE axis shows PE fluorescence, a negative control. (B) The ratio of GFP positive population shows the fraction of cells expressing GFP at a level that is higher than the background. (C) The mean fluorescence intensity (MFI) of the cells is shown as a function the ratio of tRNA to MbRS. (D) The relative level of total GFP fluorescence was calculated by multiplying the ratio and MFI of each population. 


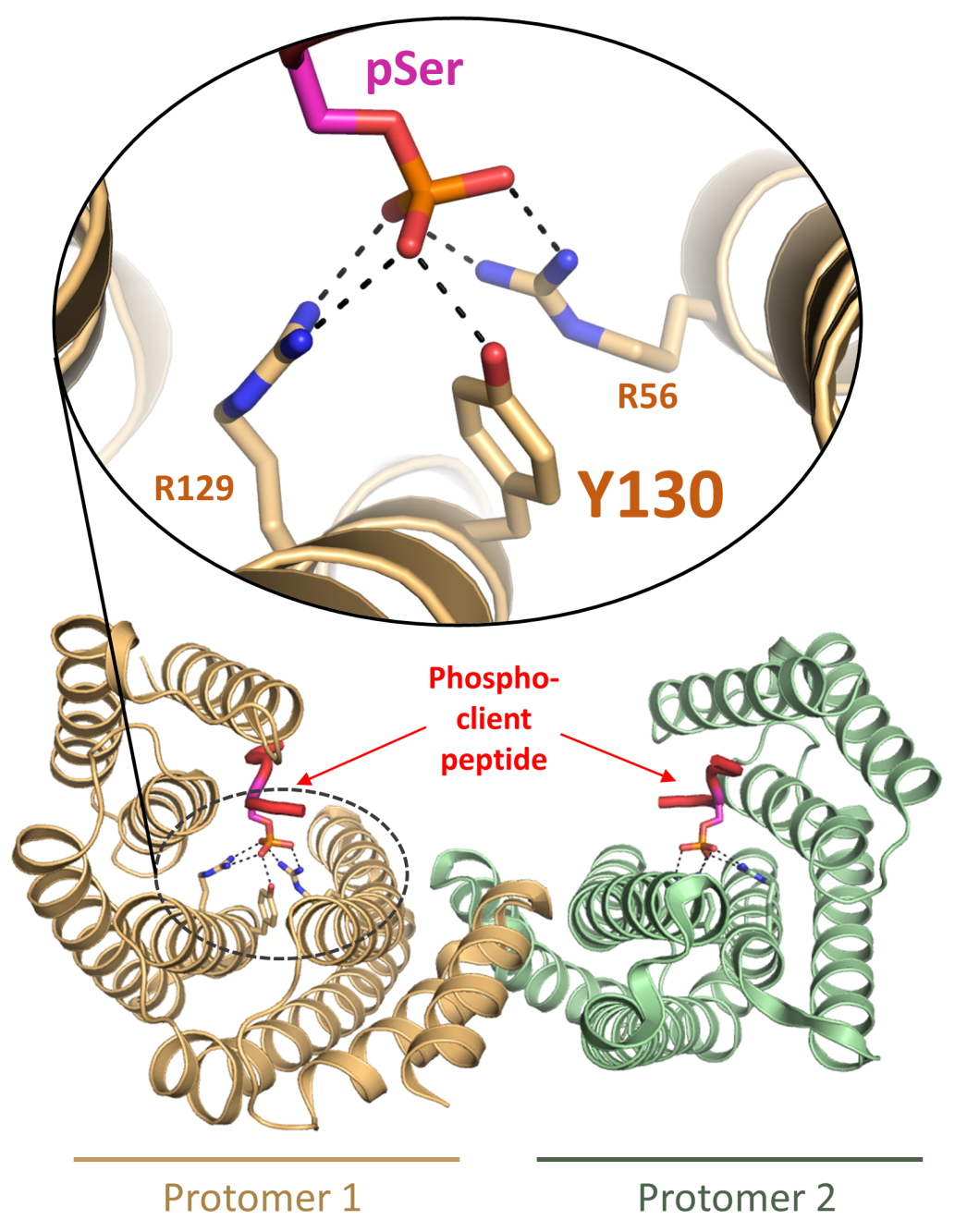

Supporting Figure 8. Tyr130 of 14-3-3 plays a key structural role in the binding of phosphorylated client proteins.

The 14-3-3 dimer (bottom) binds to phosphorylated client peptides (red cartoon) via a deep groove lined by conserved residues Y130, R56 and R129 ( $\beta$-isoform numbering) which together provide the key constellation of hydrogen bonding interactions to the phosphate of client peptide (purple carbon atoms, top inset). Given that this 14-3-3 - phosphoserine interaction is essential for client binding to 14-3-3, nitration of Tyr130 would be expected to alter client regulation by 14-3-3. PDB code 2 br9. 


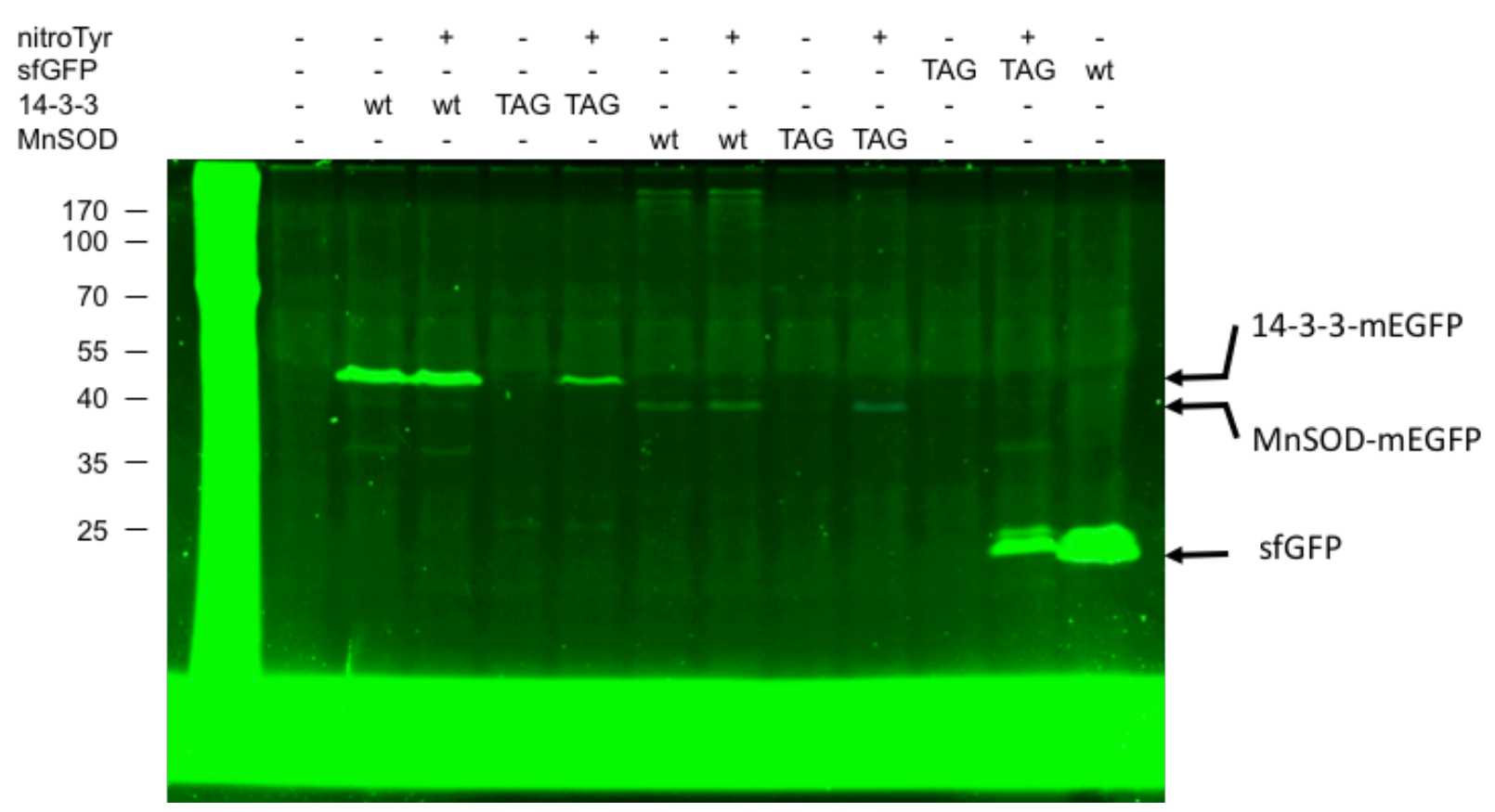

Supporting Figure 9. In gel fluorescence of HEK cell lysate demonstrating the specific GFP signal resulting from the MnSOD and 14-3-3 mEGFP fusion proteins. Full gel image of cropped images shown in figure $5 \mathrm{C}$. 


\section{Coomassie stained}

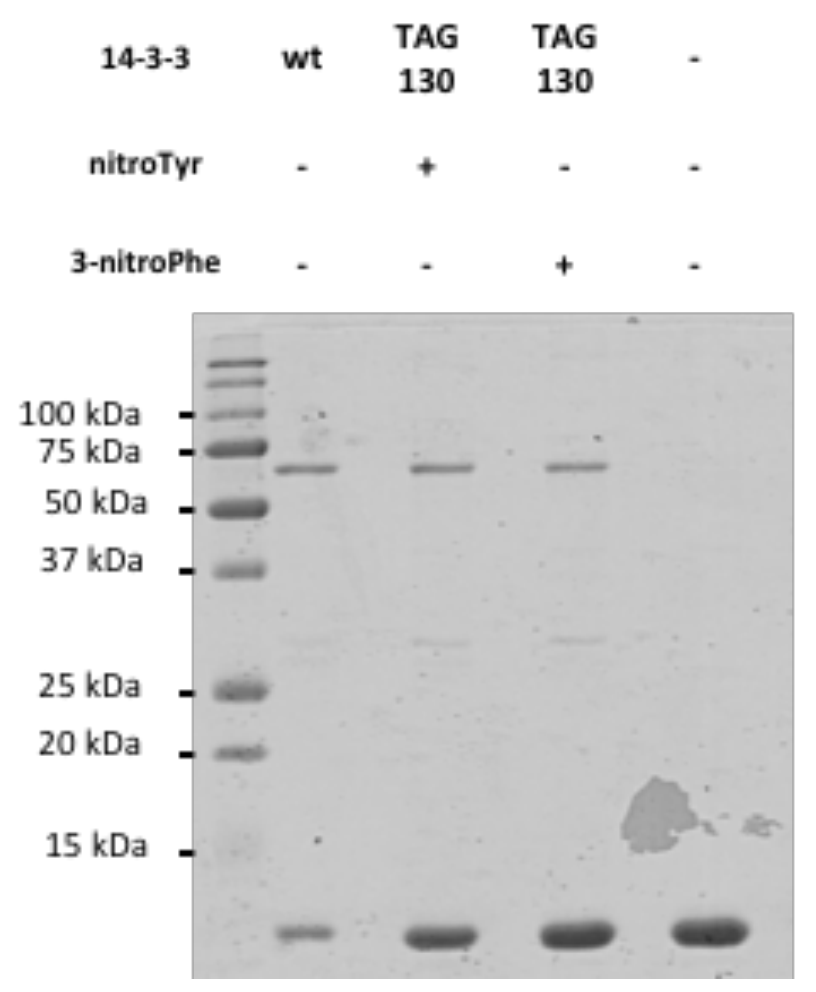

\section{Western blot}

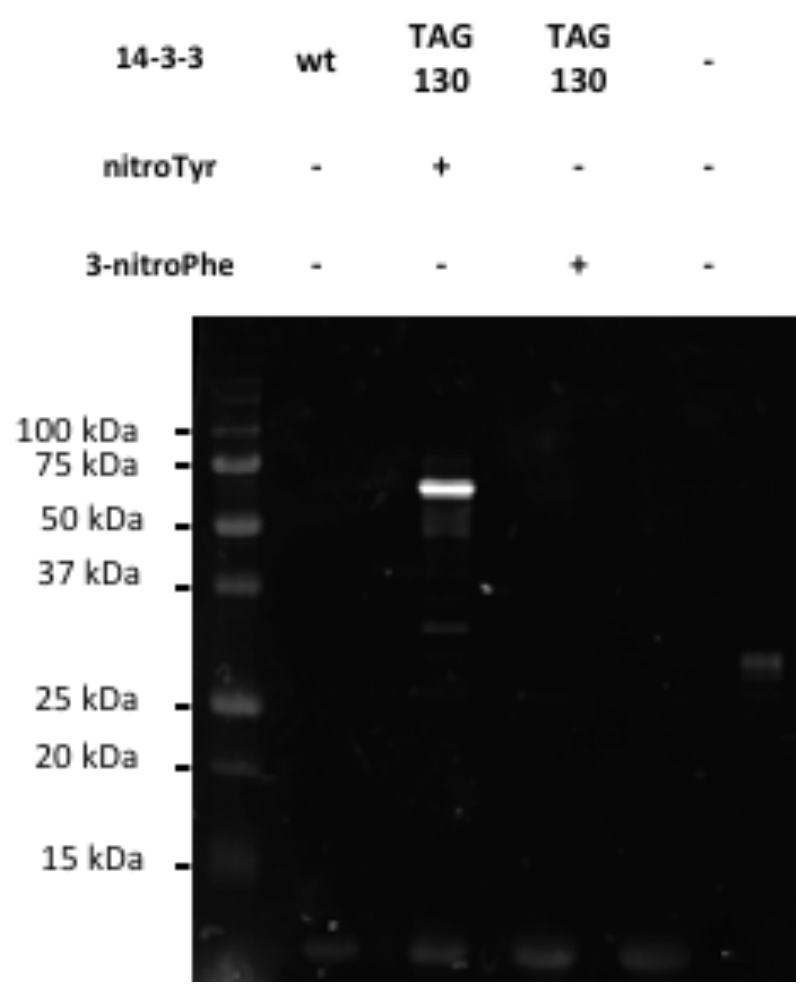

Supporting Figure 10. Characterization of nitroTyr-14-3-3-mEGFP and 3-nitroPhe-14-3-3mEGFP purified from HEK cells. Full gel images of cropped images shown in figure 5D. 